\section{Intersections}

Canadian Journal of Music

Revue canadienne de musique
Intersections CANADIAN JOURAL OF MUSIC

\title{
Pour une histoire sociale de la musique de George Crumb dans les années 1970
}

\section{Pierre Albert Castanet}

Volume 29, numéro 1, 2009

URI : https://id.erudit.org/iderudit/039108ar

DOI : https://doi.org/10.7202/039108ar

Aller au sommaire du numéro

Éditeur(s)

Canadian University Music Society / Société de musique des universités canadiennes

ISSN

1911-0146 (imprimé)

1918-512X (numérique)

Découvrir la revue

Citer cet article

Castanet, P. A. (2009). Pour une histoire sociale de la musique de George Crumb dans les années 1970. Intersections, 29(1), 17-31.

https://doi.org/10.7202/039108ar
Résumé de l'article

«Pour une histoire sociale de la musique de George Crumb dans les années 1970 » désire montrer que l'art du compositeur américain (né en 1929) reflète d'une manière kaléidoscopique les vicissitudes de la société. Usant volontiers des différentes facettes de la notion de " métaphore », l'oeuvre visionnaire de Crumb s'attache autant aux arcanes de la spiritualité qu'aux affres de la mort. Dans ce sillage, l'approche symbolique de l'écriture musicale y suit l'analyse des faits socioculturels comme des problématiques sociopolitiques du moment.
Tous droits réservés (C) Canadian University Music Society / Société de musique des universités canadiennes, 2009
Ce document est protégé par la loi sur le droit d'auteur. L'utilisation des services d'Érudit (y compris la reproduction) est assujettie à sa politique d'utilisation que vous pouvez consulter en ligne.

https://apropos.erudit.org/fr/usagers/politique-dutilisation/ 


\title{
POUR UNE HISTOIRE SOCIALE DE LA MUSIQUE DE GEORGE CRUMB DANS LES ANNÉES 1970 ${ }^{1}$
}

\author{
Pierre Albert Castanet
}

À vendre Présidents, Généraux, C.I.A, ordinateurs sans noms - Assassinats à vendre Allen Ginsberg, $\grave{A}$ vendre $\grave{A}$ vendre ${ }^{2}$

Né sous l'étoile d'un "jeudi noir ", celui du Krach boursier de 1929 (le 24 octobre) à Charleston (West Virginia - USA), George Crumb se révèle être un poète mystique, un rêveur passablement innocent. Élève du dodécaphoniste berlinois Boris Blacher, il est toujours resté hostile à la mathématique sérialisée. Dans un langage hybride, il préfère se comporter comme un artiste visionnaire et sentimentalement engagé. À l'instar d'un Luigi Nono, d'un Klaus Huber ou d'un Helmut Lachenmann... ${ }^{3}$, son geste créateur perpétue les leçons de responsabilité de l'œuvre d'art face au mal habitus de la société. La maladie du second $\mathrm{XX}^{\mathrm{e}}$ siècle (au sens étymologique de déséquilibre et de mauvais état) a ainsi impliqué un rapport sans précédent à la politique, la société, la culture, l'image et le son.

Les partitions de Crumb s'insèrent dans cette lignée d'opus dénonciateurs ou constatateurs qui prennent date vis-à-vis de ses ancêtres ou de ses contemporains qui ont lutté ou luttent pacifiquement pour une quête de salut et de vérité. Le temps des utopies est révolu, celui qui fit écrire à Madame W.-L. Landowski dans son ouvrage sur La Musique américaine - en 1952 - que le peuple américain était « idéaliste et positif, indépendant et discipliné, enthousiaste et averti, pacifique et résolu. » Le propos de cette musicologue est complété par le fait que s'il ne possède pas d'antiques archives à visiter, il a néanmoins " la légitime fierté d'être devenu l'un des principaux centres d'attraction du Monde » (Landowski 1952, p. 7). D’obédience marxiste, l'art de Crumb montre qu'au niveau du politique et de l'esthétique, « le contenu révolutionnaire et la qualité artistique tendent à coïncider» (Marcuse 1979, p. 16).

À la fin des années 1960 - à l'image des idées engagées d'un Langston Hughes (poète qui prend parti pour Martin Luther King, le Président Johnson et l'arrêt de la guerre au Vietnam, voir Hughes 1994) ou de la poésie pop d'un Allen Ginsberg (qui chante - entre autres - La Chute de l'Amérique, voir Ginsberg 1979), par exemple - ce n'est pas un hasard si, dans un cadre d'expression

1 Largement revu et augmenté, cet article émane d'une conférence prononcée à l'École des Hautes Études en Sciences Sociales (Paris) à l'occasion des Journées d'études Des ponts vers l'Amérique I. Paris, EHESS-CRAL, 7-8 décembre 2006.

2 Ginsberg 1976, p. 444.

3 Et de bien d'autres, voir Castanet 2007b, p. 3-12. 
artistique où la rébellion plus ou moins sourde reprend ses droits, huit cycles de George Crumb dont Madrigals (livres I à IV - 1965-69) ou Ancient Voices of Children (1970) exaltent sombrement et douloureusement la poésie réaliste et surréaliste de Federico Garcia Lorca (arrêté par la garde civile franquiste et fusillé en 1936 à Grenade). À ce sujet, mentionnons le quatrième mouvement d'Ancient Voices of Children intitulé Each Afternoon in Granada, a Child Dies Each Afternooon qui débute par un moment intimiste, chanté très tendrement dans une douleur sourde par la soprano. Arrive ensuite un extrait de Bist du beir mir du Petit Livre d'Anna Magdalena Bach joué par une âme d'enfant, un ange blanc, au piano-jouet, d'une manière très détachée mais pure.

Dans ce sillage faisant référence au poète espagnol sauvagement abattu, mentionnons également Night Music I (1963) (voir Lewis 1965) et Night of the Four Moons (1969). Outre Federico's Little Songs for Children (1986), la pièce de Crumb qui souligne le plus l'intensité dramatique de l'imaginaire démoniaque de Garcia Lorca est sans conteste Songs, Drones, and Refrains of Death (19621968) écrit en deux parties de structuration complexe (voir Bland 1991, p. 4) pour baryton, instruments électriques (guitare, contrebasse, piano, clavecin) et percussion. De l'avis même du compositeur (voir Crumb 2006, p. 2-3), la poésie fantastique accusant la vision mystique de la mort a stimulé et renforcé le pouvoir évocateur de l'étrange musique de Crumb. Comme dans Threnody (1938) de Paul Creston, tons de lamentation - inflexions notamment parlées - (The Guitar) et connotations sardoniques (Casida of the Dark Doves) sont de mise au sein des couplets 1 et 2 de Songs, Drones, and Refrains of Death alors qu'un sentiment de violence et de terreur envahit la cavalcade 4 du couplet 3 (Song of the Rider, 1860) incluant une "cadenza appassionata " pour deux percussionnistes agissant également avec leur voix. Enfin, pour contraster, le couplet 4 qui se présente comme une tendre berceuse lyrique et rêveuse fait référence consciemment à l'univers malherien.

À chaque instant des " refrains de mort " (termes faisant explicitement référence aux « chants et danses de la mort » de Modest Moussorgski), Crumb exploite symboliquement ${ }^{5}$ les ressources colorées de timbres sombres et de mixtures dénaturées (par l'amplification électrique mais aussi par bon nombre de parasites artificiels, extra sonores). Comme pour la courte partition de The Sleeper (1984) pour mezzo-soprano et piano bâtie d'après un texte d'Edgar Allan Poe, George Crumb aime utiliser un éventail savant de mécanismes timbriques répartis entre les différents potentiels instrumentaux afin d'accumuler des charges et libérer des décharges plus ou moins maîtrisées ou efficientes. Selon le compositeur, cet arsenal technique est proprement susceptible de suggérer le "sentiment de transcendance " (Crumb 1984, p. 2) créé par la bizarrerie d'images poétiques. On sait qu'« en art, la technique, si elle est réellement maitrise, débouche toujours sur le contraire de cette maîtrise : elle aiguise la réceptivité

4 À noter que le prototype du genre du « chant du cavalier » est représenté pour Crumb par le fameux Erlkönig de Franz Schubert.

5 William K. Bland (1991, p. 5) a relevé quelques figuralismes relatifs aux rythmes ou aux intervalles employés par Crumb. 
du sujet, la rend sensible aux tensions propres de ce qui n'est pas soi-même un sujet » (Adorno 1982, p. 337).

Dans un essai intitulé Marginalia, Edgar Allan Poe voulait justement cerner ce sentiment de surnaturalité équivoque qui exhale de certaines sphères sonores : ainsi, il dit en substance que lorsque la musique nous affecte jusqu'aux larmes, en apparence sans raison, "nous ne pleurons pas par 'excès de plaisir' mais par l'excès du chagrin irrité, impatient devant l'incapacité où nous sommes, nous simples mortels, à jouir de ces extases supranaturelles dont la musique sonore ne peut nous laisser entrevoir qu'une vision pénétrante et indéfinie » (Poe 1989, p. 1065). En fait, toute la production musicale de ce musicien niche dans ce sentiment extraordinaire d'inquiétante étrangeté peint par Poe. Surnaturel en diable, le Son crumbien semble appartenir à cette sphère sensible d'un ailleurs destructeur. "Le son en tant que seule expérience à dire, criante, éclatante, du brouillard brusquement illuminé; le son entendu, joué, compris, prophétique, chanté par des hommes, entraînant des hommes; le son, non le moyen, et pas davantage certes écrin astral, mais bien aura suprême de la réceptivité, dernière substance de l'âme, du noyau, de la latence, du symbole de Soi, sur lequel en fin de compte la musique repose; le son, phénomène supérieur de l'expression musicale et de l'idiogramme, du royaume spirituel musical » (Bloch 1977, p. 148 pour la version française), telle est la leçon que l'on retire de la Philosophie de la musique d'Ernst Bloch et qui s'applique à la lettre à l'art sonore de George Crumb.

La musique pourrait être définie comme un système de proportions au service d'une impulsion spirituelle.

\section{George Crumb (2001)}

Car c'est bien la transcendance - mue en quelque sorte en étincelles d'impureté et en matériaux accidentés - qui auréole la griffe identitaire du style de Crumb, la sonorité impure étant le seul topos métabolique de la composition pseudo narrative. Comme dit Cioran, les « vérités de tempérament doivent se payer d'une manière ou d'une autre [...] Imprégnées de subjectivité, l'on perçoit un moi derrière chacune d'elles : tout devient confession [...] jusqu'à la logique, tout lui est prétexte à autobiographie; son 'moi' a infesté les idées, son angoisse s'est convertie en critère, en unique réalité » (Cioran 1949, p. 245).

Et si les éléments de structuration formelle et les données de l'ordonnancement discursif sont souvent rigoureux dans leur simplicité même, la musique de Crumb pourrait se rapprocher de la philosophie du langage de Karl Kraus et du concept de "musique informelle » de Theodor Adorno. Car, selon le sociologue allemand, " une musique informelle serait une musique dans laquelle l'oreille perçoit, au contact vivant du matériau, ce qu'il est sorti de lui. » (Adorno 1982, p. 337). Ainsi, à méditer sur les raisons de l'art sonore de Crumb, l'écoute faussement naïve des hommes bat son plein tandis que la jouissance $\mathrm{du}$ " son sale» (voir Castanet 2007) reprend toujours ses droits. Adorno relève que "toute musique organisée de façon absolument rationnelle, où tout est réfléchi, perpétue la contrainte de la forme. Cependant qu'une musique informelle requiert également cette organisation, l'abstrusion devient pour elle 
l'avertissement de son propre caractère problématique. Elle est la tache aveugle dans laquelle se dissimule ce qui, dans le bien culturel 'musique', serait autre chose que de la culture " (Adorno 1982, p. 334).

L'approche symbolique des faits culturels ${ }^{6}$ suit de concert l'analyse des gestes politiques. Certes, comme le rappelle Samuel Butler, le monde " ne suit que ceux qui l'ont méprisé autant qu'ils l'ont servi » (2009, p. 154), mais de ce va-et-vient permanent entre le jeu et l'enjeu, entre l'histoire de l'humanité et la genèse du son, la musique fait son lit et joue son destin comme un opus bellus orienté vers ce qui, au cœur de l'expérience, est le " noyau du réel7. » - pour prendre une expression lacanienne. Comme l'a analysé Hans Belting, «l'artiste empiète sur le territoire du critique et rivalise avec l'historien. Son propre acte de représentation ressemble à celui du critique ou de l'historien et, en même temps, le contredit» (Belting 2007, p. 123). De fait, en pleine "décennie du silence $^{8}$ ", de Black Angels (1970) pour quatuor à cordes électrifié à Apparition (1979) pour soprano et piano amplifié, via les célèbres volumes de Makrokosmos (1972-73) pour piano amplifié (voir Lajoinie 1986, p. 88-101), la musique de George Crumb va refléter à sa manière les couleurs socio-politiques, emblématiques de l'histoire des États-Unis (voir Castanet 2001). À la fin des années 1960, Luciano Berio ne s'est pas privé de dénoncer la situation tragique des ÉtatsUnis en montrant que ce pays anime une idée de consumérisme extraordinaire. Dans cette situation, «...le jeune musicien doit choisir : entrer dans la société de consommation ou proposer des idées qui la contestent9.»

Illustrant des événements anciens (par exemple l'assassinat d'Abraham Lincoln pour Apparition) ou des points d'histoire immédiate (la Guerre du Vietnam pour Black Angels), les diverses pages d'écriture de Crumb vont alors se mettre au service de la métaphore de la destinée (de l'existence et de la mort). On notera qu'une des caractéristiques de l'art crumbien est que la musique pure n'existe pratiquement pas. Tous les opus sont sous-tendus par des programmes, des paraboles, des textes, des cauchemars, des songes, des références, des souvenirs... À l'instar de la philosophie anti-systématique de Hans Blumenberg, le catalogue de Crumb use volontiers des principes de la « métaphorologie $^{10} »$ (Blumenberg 1994, p. 101). Dans ce cadre original, l'image se montre comme racine de toute pensée conceptuelle, le procédé référentiel étant considéré « comme un authentique moyen de réaliser la saisie de contextes qui n'est

6 Voir Clifford 1973.

7 « Noyau " appelé parfois aussi « trou réel » par Lacan (1973, p. 63). Nous sommes loin des propos de Virgil Thomson écrivant pour une Histoire de la musique parue en 1963, que «les citoyens américains composent de la musique dans tous les styles connus. Par conséquent, on ne peut pas parler d'un style américain " (Thomson 1963, p. 1401). Pour l'anecdote, remarquons cependant que, membre de l'Académie américaine des Arts et Lettres, George Crumb est devenu en 1995 le $36^{\mathrm{e}}$ lauréat de la médaille Mac Dowell, récompense décernée annuellement par ses pairs à un artiste pour sa contribution exceptionnelle à la culture américaine.

8 Expression rapportée par Janice E. Perlman pour désigner les années 1970 aux États-Unis (Perlman 1976, p. 163).

9 «Entretien Luciano Berio - Michel Philippot », Varèse-Xenakis-Berio-Pierre Henry, La Revue Musicale $\mathrm{n}^{\circ}$ 265-266, Paris, Richard-Masse, 1969, p. 92.

10 Datant de 1960, ce terme provient des Archives d'histoire des concepts d'Erich Rothacker. 
pas réductible au noyau restreint de la métaphore absolue » (Blumenberg 1994, p. 93).

Dès lors, George Crumb fait coïncider le concept avec le symbole, l'un renforçant l'autre. Les références restent tributaires du caractère opératoire des paramètres sonores et la composition se nourrit des légendes de la nature humaine et de ses vicissitudes. La musique devient alors l'organe élémentaire du rapport aux mots et aux choses, aux signes et aux êtres, orientant sonorement et esthétiquement l'expression de la représentation du monde. Écoutez, par exemple, Star-Child (1977), sous-titré " parabole » et chanté en latin. L'écriture fait alterner figures de style desolato et expression hymnique au service du thème de l'Apocalypse. Hormis le rôle luminescent du chœur d'enfants (Gloria in excelsis; Laudemus Dominum), les dimensions conjointes du désespoir et de l'obscurité représentées par les cuivres graves, basson et contrebasson accompagnent le contrepoint de la source biblique. L'ensemble est parfois traversé de lueurs sonores rendues grâce au relief de quelques percussions tintinnabulantes (diverses cloches, cymbales antiques, glockenspiel). En dehors d'un traitement particulier du Dies Irae par le chœur d'hommes parlé (en homorythmie vocale et instrumenté sous forme de fanfare privilégiant les cuivres et les percussions), remarquons la présence sonore des chevaux de l'Apocalypse représentés par la fougue rythmique du pupitre de percussions (16 tom-toms). Fondée sur l'aura générique d'une " musique des sphères » induisant l'idée d'un continuum harmonique et la gestion d'un temps non binaire (parfois dirigé par quatre chefs non synchronisés ${ }^{11}$ ), la partition traite tout au long des sept mouvements des conditions universelles du drame humain.

En fait, le grand œuvre prophétique de George Crumb - spectateur du monde - pose des questions à résonance multiple (voir Castanet 1991, p. 1-5). Dans ce labyrinthe stylistique, la participation instrumentale ou la déclamation vocale peuvent revêtir alors des accents d'une fraîcheur puérile insoupçonnable ou se parer de susurrements sombres et nostalgiques, abordant autant le contexte de la philosophie, de la superstition, de la religion, du mystère, du bien et du mal que celui du milieu glauque contrariant et de l'aura pure étincelante, voire de la profondeur infernale et de l'illumination céleste (voir Castanet 2004, p. 1132). Considérant la nature humaine, Hans Blumenberg traite ces états de " qualités maladives». Ils seraient susceptibles de comporter "quelque chose comme une sensation aigre-douce d'un bien-être pernicieux " (Blumenberg 1994, p. 22). Pour Vladimir Jankélévitch, « la musique n'est pas seulement une ruse captivante et captieuse pour subjuguer sans violence, pour capturer tout en captivant, elle est encore une douceur qui adoucit : douce elle-même, elle rend plus doux ceux qui l'écoutent, car en chacun de nous elle pacifie les monstres de l'instinct et apprivoise les fauves de la passion » (Jankélévitch 1993, p. 10). Quant à Jean-Paul Sartre, il nomme " consciences affectives » les parts de joie, d'angoisse ou de mélancolie qui émaillent le solipsisme de tout être humain. «En un mot, les sentiments ont des intentionnalités spéciales, ils représentent

11 Le compositeur dit avoir imaginé un « contrepoint visuel » avec la chorégraphie propre à chaque chef (Crumb 2003, p. 10). 
une façon - parmi d'autres — de se transcender » (Sartre 1986, p. 137). Embrassant sans conteste les tenants sartriens - plus que jankélévitchiens - d'une "psychologie phénoménologique de l'imagination ", la palette référentielle de George Crumb tient autant du bréviaire légendaire universel que du fantasme muséologique ordinaire.

À l'orée des années 1970 et en pleine phase de "contre-culture " (Gintis 1971, p. 1400-1428), au sein de l'essor de plus en plus prégnant des conflits sociaux américains, les formes de la conscience se sont multipliées pour demander collectivement la remise en cause des valeurs fondamentales et essentielles de la société. Ainsi, « une aube nouvelle s'est levée sur l'Histoire américaine. L'empire a été vaincu au Vietnam par une guerre populaire ", relate Janice E. Perlman (1976, p. 171). Musicalement, de Luigi Nono à Jimi Hendrix, d'Henri Tomasi à Bob Dylan, de Bernd Alois Zimmermann à John Baez, de Luc Ferrari à Maxime Le Forestier, de Nguyen Thien Dao à Colette Magny..., le conflit vietnamien a inspiré bon nombre d'artistes (musiciens ou non; car on songe aussi aux poètes comme Jose Agustin Goytisolo qui a écrit en 1965 son recueil intitulé Mourir pour le Vietnam...).

Un des emblèmes compositionnels de George Crumb reste la partition de Black Angels (1970) écrite pour quatuor à cordes électrifié. Dans cet opus chambriste sous-titré " 13 images d'une nature sombre ", semblable aux modèles électrifiés des formations pop et obscène dans son essence, l'amplification systématique des instruments à cordes travaille à mettre en exergue des effets hautement surréalistes, en relation stricte avec l'environnement " psychologique " et l'atmosphère " émotionnelle " de la guerre (autant d'images sordides, autant d'affects vécus par les Américains au tournant de la décennie 1970). Précisons que deux notations non anodines figurent en bonne place sur le conducteur : d'une part on peut lire sur la première page : « in tempore belli 1970 » (ce qui veut dire : "en temps de guerre »), d'autre part, sur la dernière page est écrit : " achevé le vendredi 13 mars 1970 ».

En relation avec l'alchimie des stratégies de quête timbrique, le matériau non futile des partitions de Crumb emprunte de concert au patrimoine sonore et légendaire de l'humanité (des ravages de la pop music aux conflits internationaux les plus assassins), mais également à l'imagination débridée et à la connaissance encyclopédique du compositeur. En voie de mutation contrôlée, il louvoie singulièrement entre nature et culture. À cet effet, « le matériau n'est pas naturel, il est la synthèse de déterminations physiques mises en œuvre par les volontés croisées du luthier et du musicien, façonnées par des siècles d'exigences réévaluées et déplacées, de va-et-vient entre théorie et pratique : le matériau est synthèse culturelle », rappelle Jean-Baptiste Barrière (1986, p. 121).

Pour Black Angels, le son amplifié des instruments est parfois accompagné d'effets de cordes inhabituels (écoutez par exemple les « sons pédales» de la $D e$ vil Music); le jeu d'archet entre le sillet et la main gauche (pour produire un effet de consort de violes); des trilles sur les cordes avec un dé à coudre au bout des doigts... rappelant les pires agissements d'un Jimi Hendrix. À la manière d'un Giacinto Scelsi, George Crumb a déclaré : «Il est difficile pour moi de concevoir des sons particuliers, indépendamment de l'ensemble de l'œuvre. Un son n'est 
jamais un effet. Il doit être partie intégrante de la pièce. J'ai l'habitude d'utiliser le terme d'ethos', c'est l'œuvre toute entière qui est dans le son » (Crumb 2001). De plus, dans Ancient Voices of Children, le compositeur ne désirerait-il pas accroître « l'intensité expressive » en juxtaposant une « suggestion de Flamenco » avec une citation de Jean-Sébastien Bach ou une réminiscence de Gustav Mahler avec un «souffle d'Orient » (Castanet 2007, p. 350) ?

Nous l'avons vu, cette pièce pour quatuor électrifié montre une fenêtre ouverte sur les troubles «surréalistes » de la guerre américaine. Auréolée par les distorsions électriques, la parasitose sonore dénonce un univers chagrin, malsain, impur et sali à dessein. Intermédiaires entre la terre et Dieu, les quartettistes doivent jouer des maracas, tam-tams et autres water-tunes. Ils ont également à manier des sifflets, à chuchoter et à crier. En fait, « la violence n'est peut-être pas le moyen le plus efficace d'étrangler l'expression, remarque Vladimir Jankélévitch, car il y a dans ses fureurs mêmes et dans ses outrances quelque chose de suspect qui annonce l'intention passionnée et le tourment, et l'humaine, trop humaine angoisse » (1983, p. 57, extrait de " Ne rien exprimer : indifférence affectée $»)$.

Néanmoins, à l'époque, pour Crumb, « tout était sens dessus dessous. Des choses terribles flottaient dans l'air... ils trouvèrent leur voie vers les Anges Noirs » (Crumb 1996, p. 54). En l'occurrence, la pièce ${ }^{\circ} 7$ située au strict milieu du cycle condense un thrène - un chant de deuil ${ }^{12}$ - en hommage à ces créatures maléfiques, l'image de "l'ange noir » restant un moyen conventionnellement utilisé pour symboliser l'ange déchu, ayant perdu toute superbe et tout rayonnement. Car si la symbolique de l'ange candide promeut la pureté d'un ordre hiérarchique spirituel, celle de l'ange noir veut contrecarrer le message divin et faire des signes avant-coureurs du Sacré, une constatation du malheur terrestre. "En vérité, les liens entre l'appréciation réfléchie de la musique sérieuse, des beaux-arts, de la littérature, d'un côté, la conduite politique, de l'autre, sont tellement obliques qu'ils nourrissent le soupçon que la grande culture, loin de faire halte à la barbarie, peut lui donner un zeste et un vernis particuliers ", remarque George Steiner (1997, p. 301).

Lopus crumbien a été conçu comme une "parabole de notre monde contemporain tourmenté ». Les nombreuses allusions quasi-programmatiques de la partition sont extrêmement symboliques, "bien que la polarité essentielle - Dieu contre Satan - implique plus qu'une simple réalité métaphysique " (Crumb 1990), explique le compositeur. De plus, à l'évidence antipropagandiste, cette pièce moderniste est à considérer - de l'aveu même du compositeur - comme un «testament humain » (Starobin 2003). D’obédience joycienne, la structure en arche veut signifier le voyage d'une âme humaine dans un parcours retraçant l'envol, l'absence et le retour comme trois stations à entrées plurielles respectivement liées à la Grâce ou à la Bénédicité perdue, à l'Annihilation spirituelle et à l'Espoir ou la Rédemption. Comme souvent dans ses partitions, « le musicien ouvre la cage aux chiffres » (Cocteau 1979, p. 45)

12 Ce thrène développe un jeu de densité allant de l'unisson joué piano à la texture en quatuor exécutée triple forte, le graphisme faisant penser à une étude sur les arborescences xénakiennes. 
pour ce transport balisé en trois étapes (transport entendu au sens premier de métaphore).

En effet, ritualisé à souhait, un symbolisme numérologique des 7 et $13-$ cryptogrammes fatidiques - habille tous les paramètres (hauteurs, durées, schémas de répétitions). Entre chiffrage rudimentaire et déchiffrement complexe, George Crumb tisse un réseau de connotations à valeur rhétorique (au sens où les figures numériques tendent à rendre plus exaltante l'expression de la pensée). L'ars rhetorica crumbien induit une logique discursive liée à la réception (voire à la compréhension ou au déchiffrement) de l'auditoire. Dans ce cadre, la technique de la citation peut être un des moyens rhétoriques qui implique fortement l'auditeur (voir Pasticci 2005, p. 103-107). En 1964, Roland Barthes a écrit que "déchiffrer les signes du monde, cela veut toujours dire lutter avec une certaine innocence des objets » (Barthes 1985, p. 228). À certains endroits de la partition de Black Angels, ces chiffres babéliques sont à compter rituellement à haute voix en différentes langues (dont l'allemand, le français, le russe, le hongrois, le japonais, le swahili). Entre l'énoncé compris et la formule incompréhensive, les éléments crypto-dramatiques d'une maïeutique intense sont petit à petit révélés car, comme l'explique Michel Imberty, « le sens naît de la tension entre la signification usuelle latente et la stylisation qui déguise cette signification par le contexte » (Imberty 1981, p. 16).

La figure du Diable est présente au niveau de l'intervalle de triton (diabolus in musica) ainsi que dans la figure stylistique du trille, faisant référence au célèbre Trillo di diavolo de Tartini. Celle de Dieu sera confortée par des effets de pureté d'harmonica de verre (jeu d'archet sur une série de verres en cristal accordés modalement et par un fond en si bémol majeur). Il existe plusieurs allusions à la musique tonale : une citation du quatuor de Franz Schubert La Jeune fille et la mort, une Pavane Lachrymae, une Sarabande... Sans parler des références à la séquence latine du Dies Irae (signifiant « Jour de colère »). Cette musique virtuose du Diable qui est confiée principalement au violon 1 jouant le personnage de la «Vox Diaboli » est suivie d'une Danse macabre "grotesque » et "satirique ». Accompagnée par un trio jouant parfois de la percussion, la "Musique du Diable » aura son pendant dans la "Musique de Dieu», calme et sereine, interprétée par le violoncelle solo, accompagné par un trio homorythmique de verres en cristal. Traitée comme un pamphlet satirique, la " Danse macabre » qui suit demandera le complément de sifflet et de maracas.

Jouant sur la phénoménologie de l'intertextualité, l'art sonore de Crumb verse, grâce notamment au geste citationnel, dans la catégorie postmoderne ${ }^{13}$. Hybridé par maints réseaux métaphoriques, il devient "le symptôme engagé d'une musique qui souhaite briser l'interdiction du souvenir " (RamautChevassus 1998, p. 44).

Sous-titré "Chants et vocalises élégiaques ", Apparition (1979) pour voix et piano amplifié est écrit sur un texte de Walt Whitman. C'est la première partition de Crumb pour voix soliste et piano. Hormis quelques exercices de jeunesse, c'est aussi sa première mise en musique d'un texte en anglais. Le texte

13 Voir Weber 1989. 
est extrait de "When Lilacs Last in the Dooryard Bloom'd », poème intégré à un recueil intitulé Memories of President Lincoln (commémorations du Président Lincoln). L'auteur a en effet écrit ses vers juste après l'assassinat d'Abraham Lincoln, le 14 avril 1865. Bien que le poème soit conçu comme une élégie ${ }^{14}$ funèbre, Crumb a choisi la majeure partie de son texte dans une portion soustitrée « Death Carol » (Chant de la Mort). Si elle constitue une parenthèse dans les références directes au président défunt, elle met en revanche à l'honneur une écriture imaginative qui se penche sur l'expérience mortuaire humaine. « Là où le poème gênait les rondes musicales, l'exégèse fidèle peut les élargir, elle peut, réveillant la mémoire et s'éloignant du simple commentaire, les dessiner sur la carte des archétypes ", note Ernst Bloch (1977, p. 148). Il ne s'agit pas ici de peindre sonorement la personnalité d'un président de la république en pleine guerre de Sécession - comme a pu le faire Aaron Copland en 1942 avec Lincoln Portrait écrit pour récitant et orchestre - mais de composer une élégie commémorative aux accents tantôt rebelles, tantôt recueillis.

Dans Apparition, au travers de six mouvements entrecoupés de trois vocalises, texte et musique se polarisent sur des métaphores concises et denses de la présence et de l'absence. Le cycle crumbien est au fond rassurant car la fin de la vie n'est jamais décrite comme la mort véritable. Au contraire, elle est cyclique (le $1^{\mathrm{er}}$ mouvement est semblable au dernier et porte le même titre : The Night Silence under Many a Star). La musique montre que la vie est toujours en perpétuel état de recommencement. Comme dans Black Angels, les forces de l'esprit aspirent à un retour enrichissant vers une force vitale universelle. La pièce est donc placée sous l'égide de symboles d'éternité : " la nuit », symbole de l'univers, "le rivage de l'océan », symbole du mouvement et du temps; " l'âme ", symbole de la conscience; le " corps se blottissant contre soi, symbole du cycle de la vie et de la mort. " La conscience de la non-vie comme émancipation fait partie des espoirs les plus mémorables de l'homme. La métaphore de Whitman présente ainsi la mort sous les traits signalétiques d'un corps féminin, l'allégorie étant investie du pouvoir de renaissance. Une fois de plus, ce concept renoue avec l'idée puissante d'un sauveur rédempteur. Pour illustrer cette idée forte, Crumb utilise dans Approach Strong Deliveress! des accents de marche implacable. Propulsée par une énergie impitoyable, ce quatrième mouvement d'Apparition se veut serein à la fois par sa part d'espoir et par l'acceptation non douloureuse de la perte de l'existence.

La part métaphysique de l'art de Crumb s'attache à la représentation héraclitéenne de l'humanité, montrant que les couleurs tragiques de la destinée se reflètent inlassablement dans la réalité de "la cruauté naturelle des choses ", comme dit Nietzsche (1964, p. 121). Au reste, Hugues Dufourt rappelle que, pour ce philosophe allemand, la musique est « une science intuitive qui, dans le ravissement horrifié et le désespoir absolu, nous procure la jouissance de l'éternité et la vision tragique du monde » (Dufourt 1991, p. 42). La philosophie

14 On pense à l'Elegy for JFK (1964) composée par Igor Stravinski juste après l'assassinat de John Fitzgerald Kennedy, à Dallas ou encore à A Child of Our Time (1944) de Michael Tippett qui évoque le meurtre, en 1938, d'un diplomate de l'ambassade d'Allemagne à Paris par un jeune réfugié juif et qui cerne les pogromes qui s'ensuivirent dans l'Allemagne hitlérienne. 
compositionnelle de George Crumb incarnerait une forme de " conscience imageante » (Sartre 1986, p. 37) se comportant à la fois d'une manière représentative, objectale, réflexive, perceptive, spontanée, intuitive, prospective, affective, sensible et créatrice. "D'ailleurs, l'image, comme le signe, est une conscience. Il ne saurait être question d'un lien extrinsèque entre ces deux consciences [...] Une conscience est tout entière synthèse, tout entière intime à elle-même : c'est au plus profond de cette intériorité synthétique qu'elle peut se joindre, par un acte de rétention ou de protention, à une conscience antérieure ou postérieure », analyse Jean-Paul Sartre (1986, p. 57).

" Dark Mother » extrait d'Apparition se présente comme une élégie révérencielle, une manière de prière calme où la vision extatique du monde laisse poindre un espoir instinctif religieux : l'image de la mort comme délivrance. Cette pièce est suivie de Vocalise 2 intitulée "invocation ». Il s'agit d'une cadence dramatique et virtuose qui désire se rapporter aux représentations maléfiques de la nature. "Une conscience imageante comprend un savoir, des intentions, peut comprendre des mots et des jugements ", relève Sartre (1986, p. 187). Parfois les mots sont des chiffres (comme dans Black Angels), parfois, ils sont asémantiques (comme dans Apparition). En tous les cas, croyant au pouvoir mystérieux du « mana ", Crumb pourrait affirmer avec Aragon qu' il y a des mots qui possèdent pendant un temps variable une puissance incantatoire " (Aragon 1929, p. 57) - ce qu'Édouard Glissant cerne comme étant la « Poétique de la relation» (Glissant 1990).

Chez le musicien américain, le "savoir pur» (Sartre 1986, p. 202) tiendrait alors de la "conscience " de la règle scolastique (des «beautés de convention " comme disait Jean-Jacques Rousseau, telles des formes à refrains ou plus largement des hommages ostentatoires ou masqués aux anciens), le « savoir impur » étant alors redevable de la " conscience " hors la loi (hybridation des formes, instrumentarium hétéroclite, œuvre manifeste, aura d'un geste d'engagement sociopolitique...) - voire de l'inconscience.

La tradition se travestit d'époque en époque,

mais le public connaît mal son regard et ne la retrouve jamais sous ses masques.

Jean Cocteau, Le coq et l'arlequin (1979, p. 70)

Par ailleurs, le geste compositionnel crumbien tend à dénoncer cette attitude portée à la psychologie historique de l'expression humaine, délimitée à maintes reprises par les écrits méthodologiques d'Aby Warburg (voir Warburg 1990), puis d'une certaine manière par les travaux d'analyse iconologique signés par Erwin Panofsky. En fait, il s'agit surtout d'approcher et de comprendre la cohésion des méga-processus évolutifs qui gouvernent la teneur des couleurs stylistiques, c'est-à-dire les différentes transformations de la structure expressive engagée, et les diverses métamorphoses de la fonction représentative de l'œuvre d'art musicale dans une société donnée. Ainsi, loin de se limiter comme Panofsky à l'identification de la forme en vue d'accéder au contenu représenté, Warburg oriente par exemple le truchement explicatif vers l'élucidation des 
tensions et des luttes dont l'appareil formel figure l'objectivation, la manifestation ou l'issue.

En l'occurrence, l'acte artistique de formalisme crumbien - à la fois structurel, matiériste, timbrique mais également contestataire, rituel - permet de rechercher consciemment des médiations ombrageuses entre les schèmes symboliques définissant un mode figuraliste de représentation sémantique de l'œuvre musicale (portées suggestives, sous-titres éloquents, programmes explicatifs, paratextes omniprésents) et une enveloppe informative contextuelle corollaire (la métaphore nocturne, l'appel de l'au-delà, la fatalité, l'expérience de la mort, l'aura de la guerre, le désespoir et la renaissance...). Entre le clair et l'obscur, entre l'adret et l'ubac, Crumb se réfugie la plupart du temps dans l'ombre. Cette notion d'ombre - comme dirait Roland Barthes - « c'est un peu d'idéologie, un peu de représentation, un peu de sujet : fantômes, poches, traînées, nuages nécessaires : la subversion doit produire son propre clairobscur» (Castanet 2002, p. 38).

Or, le plan de subversion de George Crumb (tout comme celui d'Helmut Lachenmann) tient dans la technique d'écriture. Theodor Adorno a écrit que « la protestation de l'art contre la culture dominante ne peut prendre de force qu'à travers la technique " (Adorno 1982, p. 240). Celle de Crumb s'articule volontiers autour de la façon de dénaturer le matériau au service de l'idée, d'où une musique objectale qui sort de l'ordinaire et qui se comporte singulièrement. «Elle devient à la fois plus intransigeante, du fait d'une impitoyable complexité qui découle du principe de construction, et plus urbaine » (Adorno 1982, p. 240), conclut le philosophe allemand. Par ailleurs, Hans Blumenberg note que la métaphore constitue d'abord un " dérangement » et qu'elle peut s'apparenter au concept d'" anticoncordance " d'Edmund Husserl (Blumenberg 1994, 94-95). Pour d'autres, la métaphore se nomme " collision » (Friedrich Hegel), « illusion » (Gaston Bachelard), « subversion » (Edmond Jabès), « fiction » (Jorge Luis Borges), " apparition » (Paul Virilio), " mirage » (Jean-Claude Risset), " catastrophe » (René Thom), « énigme " (Thomas S. Kuhn), «événement » ou « lapsus » (Daniel Siboni), «blessure » (Helmut Lachenmann), " rature » (Michael Levinas), "fêlure » (Georges Bataille), " accident » (Francis Bacon, Paul Ricœur, Michael Levinas), " anomalie » (Sophie Stévance) ${ }^{15} . .$.

Comme le remarque Hans Blumenberg, "le corps étranger exotique est qualifié de 'simple métaphore' par un acte d'auto-affirmation : le dérangement devient support. Dans l'expérience cela correspond à la nécessité d'incorporer l'événement le plus surprenant qui survient à la limite du prétendu 'miracle' dans le système général de causalité » (Blumenberg 1994, p. 95).

La musique de demain sera la fille des musiques d'hier et d'aujourd'hui, même si c'est une enfant rebelle.

George Crumb (2001)

Cependant, nous ajouterons que si l'art de Crumb déchire parfois les règles de la normalité ancestrale et de la bienséance langagière, il se réalise toujours

15 Voir Castanet 2007, p. 365-366, et Castanet 1995, p. 289-305. 
loin des abîmes de l'abstraction gratuite et décharnée (en fait - pour parler quasi à la manière d'Edmund Husserl - l'anormalité, en tant que rupture de l'unité du phénomène à l'origine cohérent, est intégrée dans une normalité supérieure »). Ainsi, au travers de cette optique pragmatique de l'efficacité sonore - parfois énigmatique - le compositeur américain semble montrer que l'humanité a toujours besoin de sens et d'images dérivées, de questionnements et de stimuli corollaires pour comprendre et cerner les arcanes du monde (futil nouveau), pour y percevoir sa propre figuration et cristalliser les faisceaux unitaires de son accomplissement spirituel.

Concernant le parfum spirituel qui exhale des différents livres de Makrokosmos, Vincent Lajoinie a distingué quatre tendances dominantes : le "pôle chrétien » illustré par Agnus Dei (II/12, Capricorne) ou Crucifixus, (I/4, Capricorne), émaillé d'intervalles de tritons... le " pôle cosmique » représenté entre autres par The Magic Circle of Infinity (Moto Perpetuo) (I/8, Lion) ou Spiral Galaxy (I/12, Verseau)... le " pôle païen » regroupant The Mystic Chord (II/2, Sagittaire), Voices from "Corona Borealis " (II/10, Verseau)... et le " pôle diabolique »- antithèse obligée - dans lequel s'expriment The Phantom Gondolier (I/5, Scorpion), Ghost-Nocturne : for the Druids of Stonehenge (Night-Spell II) (II/5, Vierge). Le musicologue conclut en disant que « de cette profusion œcuménique, il est naturellement impossible de tirer le moindre indice en ce qui concerne la religion de l'auteur » (Lajoinie 1986, 92-93).

La contemporanéité s'inscrit, en fait, dans le présent en le signalant avant tout comme archaïque, et seul celui qui perçoit dans les choses les plus modernes et les plus récentes les indices ou la signature de l'archaïsme peut être un contemporain. Giorgio Agamben, Qu'est-ce que le contemporain? (2008, p. 33)

À propos du sens véhiculé par les partitions graphiques ou par le contenu spirituel de certains opus crumbiens, citons encore Hans Blumenberg qui écrit que «les métaphores sont des fossiles-conducteurs issus d'une couche archaïque du processus de la curiosité théorique, qui n'est pas forcément anachronique sous prétexte qu'on ne peut revenir à la profusion de ses stimulations et attentes de vérité » (Blumenberg 1994, p. 94). Au reste, à l'instar d'un Hugues Dufourt par exemple, George Crumb reste un musicien saturnien qui broie perpétuellement le noir de la mélancolie, qui se complait dans les profondeurs de la mort, qui dépeint le lugubre avec nécessité, et qui habite les ombres errantes par nature plus que par envie. "La métaphorologie, si elle ne veut pas se limiter à la performance de la métaphore pour la formation des concepts, mais l'utiliser comme fil conducteur du regard porté sur le monde de la vie, ne pourra éviter de s'intégrer dans l'horizon plus vaste d'une théorie de l'inconceptuabilité » (Blumenberg 1994, p. 101).

Il n'empêche, entre la conscience et l'imagination, entre le dicible et l'indicible, entre la fable et l'ineffable, entre un sentiment permanent de douce persécution et une "illusion d'immanence " (Sartre 1986, p. 17 et 123) de simulacres et de sortilèges... l'art de George Crumb - allant du modèle pop à l'extravagance sonore, de la pseudo prière à la danse macabre, du chant de glorification à l'in- 
vocation maléfique, des souvenirs solennels de l'histoire américaine à la dénonciation testamentaire des conflits mondiaux - ne se présente-t-il pas simplement comme le mixte d'un récit existentiel en sa tour de Babel, celui de notre vie singulièrement imaginative et de notre propre douleur quotidienne?

\section{RÉFÉRENCES}

Adorno, Theodor W. 1982. Quasi una fantasia. Paris : Gallimard.

Agamben, Giorgio. 2008. Qu'est-ce que le contemporain ? Paris : Rivages poche / Petite Bibliothèque.

Aragon, Louis. 1929. "Introduction à 1930 ». La révolution surréaliste, $\mathrm{n}^{\circ} 12$ (15 décembre).

Barrière, Jean-Baptiste. 1986. " Mutations du matériau, mutations de l'écriture ». InHarmoniques $\mathrm{n}^{\circ} 1$ (décembre), 118-124.

Barthes, Roland. 1985. «Cuisine du sens». Dans L'aventure sémiologique. Paris : Seuil.

Belting, Hans. 2007. L'histoire de l'art est-elle finie ? Paris : Gallimard.

Bland, William K. 1991. «Songs, Drones, and Refrains of Death», livret du CD Bridge Records BCD 9028.

Bloch, Ernst. 1977. L'esprit de l'utopie. Paris : Gallimard.

Blumenberg, Hans. 1994. Naufrage avec spectateur, Paradigme d'une métaphore de l'existence. Paris : L'Arche.

Butler, Samuel. 2009. Contrevérités. Paris : Payot \& Rivages.

Castanet, Pierre Albert. 1991. À propos de Four Nocturnes, Processional, Black Angels, Ancient Voices of Children de George Crumb. Paris : L'Itinéraire. - 1995. "Le rituel, la métaphore et la métamorphose ». Dans Hugues Dufourt - 25 ans de musique contemporaine. Paris : Michel de Maule, p. 289305.

—. 2001. "Éthique et esthétique de la musique de George Crumb», conférence prononcée le 8 mars 2001. Conservatoire National de Région de Caen, $19^{\mathrm{e}}$ festival Aspects des musiques d'aujourd'hui.

—. 2002. "Les passeurs d'ombre de la musique contemporaine ». Dans La manière noire. Paris : Michel de Maule.

—. 2004. «Un kaléidoscope fin de siècle ». Dans Histoire de la musique, sous la dir. de Marie-Claire Beltrando-Patier. Paris : Bordas.

- 2007. Tout est bruit pour qui a peur - Pour une histoire sociale du son sale. Paris : Michel de Maule. (Publ. orig. 1999)

— 2007b. "Pour une quête poétique de salut et de vérité - Béla Bartók, Viktor Ullmann, George Crumb ». Programme de la Philharmonie du Luxembourg, 20 avril 2007. Luxembourg.

Cioran, Émile M. 1949. "Vérités de tempérament ». Dans Précis de décomposition. Paris : Tel Gallimard.

Clifford, Geertz. 1973. The Interpretation of Cultures. New York : Basic Books. Cocteau, Jean. 1979. Le coq et l'arlequin. Paris : Stock Musique.

Crumb, George. 1984. "Program note », The Sleeper. New York : Peters.

—. 1990. Livret du CD Elektra Nonesuch 7559-79242-2. 
1996. Programme de Musica 96. Strasbourg : Musica.

- 2001. Échange suite à une conférence (voir Castanet 2001). Conservatoire National de Région de Caen, $19^{\mathrm{e}}$ festival Aspects des musiques d'aujourd'hui.

- 2003. "Star-Child». Livret du CD Bridge Records 9174.

- 2006. "Songs, Drones, and Refrains of Death ». Livret du CD Naxos 8.559290 .

Dufourt, Hugues. 1991. « La tragédie de la musique enfantée par l'esprit du nihilisme ». Dans Musique, pouvoir, écriture. Paris : Christian Bourgois.

Ginsberg, Allen. 1976. "Les États-Unis en questions ». Les Temps Modernes, $\mathrm{n}^{\circ}$ 361-362 (août-septembre), 444-447. Traduit par J.-J. Lebel.

1979. La chute de l'Amérique. Paris : Flammarion.

Gintis, Herb . 1971. «Contre-culture et militantisme politique ». Les Temps Modernes, n²95 (février), 1400-1428.

Glissant, Édouard. 1990. Poétique de la relation. Paris : Gallimard.

Hughes, Langston. 1994. The Collected Poems. New York : Vintage.

Imberty, Michel. 1981. Les écritures du temps. Paris : Dunod, Bordas.

Jankélévitch, Vladimir. 1993. La musique et l'ineffable. Paris : Seuil.

Lacan, Jacques. 1973. Les quatre concepts fondamentaux de la psychanalyse. Paris : Seuil, coll. Points-Essais $n^{\circ} 217$.

Lajoinie, Vincent. 1986. «Makrokosmos de George Crumb : 24 pièces en quête d'auteur ». Contrechamps n6. Lausanne : L'Âge d'Homme.

Landowski, Wanda L. 1952. "Avant-propos ». Dans La musique américaine. Paris : Pierre Horay-Flore.

Lewis, Robert Hall. 1965. « George Crumb: Night Music I . Perspectives of New Music 3, $\mathrm{n}^{\circ} 4,143-151$.

Marcuse, Herbert. 1979. La dimension esthétique - Pour une critique de l'esthétique marxiste. Paris : Seuil.

Nietzsche, Friedrich. 1964. La naissance de la tragédie. Genève : Gonthier, Bibliothèque Médiations.

Pasticci, Suzanna. 2005. "Rhétorique et citation dans la musique du XX siècle ». Musurgia, vol. 7, n 1-2, 99-107.

Perlman, Janice E. 1976. « Les groupes de base aux États-Unis dans les années soixante-dix », dans "Les États-Unis en questions ». Les Temps Modernes, $\mathrm{n}^{\circ}$ 361-362 (août-septembre), 163-186.

Poe, Edgar A. 1989. « Marginalia ». Dans Contes, essais, poèmes. Paris : Robert Laffont, coll. Bouquins.

Ramaut-Chevassus, Béatrice. 1998. Musique et postmodernité. Paris : Presses Universitaires de France, coll. Que sais-je n 3378.

Rothacker, Eric. 1960. "Paradigmen zu einer Metaphorologie ». Archiv für Begriffsgeschichte, $\mathrm{n}^{\circ} 6$.

Sartre, Jean-Paul. 1986. L'imaginaire - Psychologie phénoménologique de l'imagination. Paris : Gallimard.

Starobin, David. 2003. "Unto the Hills; Black Angels ». Livret du CD Bridge Records 9139.

Steiner, George. 1997. Passions impunies. Paris : Gallimard. 
Thomson, Virgil. 1963. «La musique américaine ». Dans Histoire de la musique II, vol.2, sous la dir. de Roland Manuel. Paris : Gallimard, 1963.

Warburg, Aby. 1990. Essais florentins. Paris : Klincksieck.

Weber, Horst. 1989. « George Crumb: Amplified Piano - Amplified Tradition. Zur Kritik 'Postmodern' Komponierens ». Dans Das Projekt Moderne und die Postmoderne (sous la dir. de W. Gruhn). Regensburg: Gustav Bosse Verlag.

\section{RÉSUMÉ}

"Pour une histoire sociale de la musique de George Crumb dans les années 1970 » désire montrer que l'art du compositeur américain (né en 1929) reflète d'une manière kaléidoscopique les vicissitudes de la société. Usant volontiers des différentes facettes de la notion de "métaphore ", l'œuvre visionnaire de Crumb s'attache autant aux arcanes de la spiritualité qu’aux affres de la mort. Dans ce sillage, l'approche symbolique de l'écriture musicale y suit l'analyse des faits socioculturels comme des problématiques sociopolitiques du moment.

\footnotetext{
ABSTRACT

“Towards a Social History of George Crumb's Music in the 1970s" wishes to show that the art of this American composer (b. 1929) reflects in a kaleidoscopic manner the changing nature of society. By readily applying various facets of the notion of "metaphor," Crumb's visionary output relates as much to the mysteries of spirituality as to the throes of death. Thus, the symbolic approach to musical composition follows the analysis of socio-cultural realities as well as the socio-political circumstances of the time.
} 